\title{
Mechanically driven active and passive grains as models for egress dynamics
}

\author{
Mahdieh Mohammadi', Kirsten Harth ${ }^{1}$, Dmitry Puzyrev ${ }^{1}$, , Torsten Trittel ${ }^{1,}$, Tina Hanselka ${ }^{1}$, and Ralf \\ Stannarius ${ }^{1, *}$ \\ ${ }^{1}$ Institute of Physics, Otto von Guericke University, Magdeburg, Germany
}

\begin{abstract}
Passages of people or cattle through narrow entrances or exits occur in manifold situations. They are difficult to study experimentally, because one has to carefully separate objective, physical parameters from subjective, individual motivations, manners and temperament. Mechanically excited physical model systems can help to discriminate some of these classes of parameters. We characterize active and passive particles of equal shape and mass on a vibrating plate and study their bottleneck passage dynamics. They show fundamentally different scaling behavior.
\end{abstract}

\section{Introduction}

Vibration-driven granular particles have been studied in a variety of experiments, e. g. [1-13]. In many of these, the excitation leads to a diffusive motion of the individual particles. In the context of the present study, we will refer to these as passive grains. They do not have a preferential direction of motion, even though the diffusive motion can be anisotropic if the particle shape is not isotropic in the plane. Some experiments have been performed with particles that either possess a preferential sense of rotation [10], or a preferential direction of motion $[2,7,8,12]$. Such a directed motion can be achieved by breaking the back-front symmetry of such particles, e.g., by a polar shape of the particle, like tapered ends of rod-like objects or asymmetric brushes in contact with the bottom plate, or by an asymmetric distribution of the mass inside the particle, or a combination of these. With such self-propelled grains, it is possible to simulate features of biological systems experimentally in vitro. Reviews of such studies can be found, e. g. in Refs. [14, 15].

This paper describes the preparation of grains with tuned propagation characteristics and their dynamic behavior in different geometries. An important difference to earlier studies is that we break the in-plane isotropy of the setup by a slight tilt of the vibrating plate by few degrees. Thus, we can force the ensembles to drift in a predefined direction, in addition to their individual particle motions. This setup allows, for example, to study sedimentation processes in active granular matter. Another qualitative difference to earlier investigations is that we can compare grains with identical shapes and mass, so that steric

*e-mail: ralf.stannarius@ovgu.de

A video is available at https://doi.org/10.48448/66v8-4b62

(C) The Authors, published by EDP Sciences. This is an open access article distributed under the terms of the Creative Commons Attribution License 4.0 (http://creativecommons.org/licenses/by/4.0/).

interactions will be the same for all particles, and their activity is the crucial parameter.

\section{Particles and experimental setup}

a)

b)
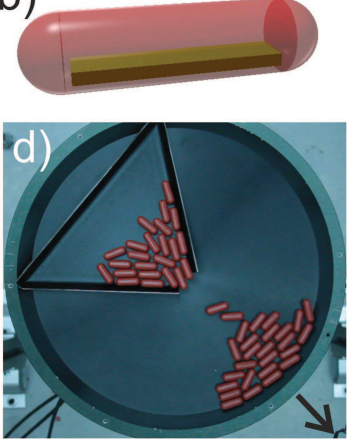

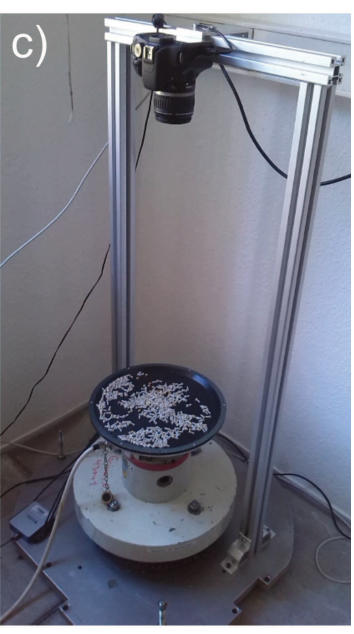

Figure 1. a) Active and b) passive capsules used in the experiments. Their length is $21 \mathrm{~mm}$, the diameter is $7 \mathrm{~mm}$ (aspect ratio 3). c) shows the setup with shaker, ground plate and camera. d) is the top view of the vibrating plate with the triangular $2 \mathrm{D}$ silo during discharge. The tilt direction is marked by a black arrow.

Figures $1 \mathrm{a}$ and $1 \mathrm{~b}$ show the particles used in the experiments, pharmaceutical pills that are either asymmetrically loaded by a $0.48 \mathrm{~g}$ lead bullet (air rifle ammunition) (a) or symmetrically loaded with a metal bar of the same weight (b). Details are described in Ref. [16]. The grains are excited by a vibrating plate mounted on an electromechanic shaker 
(Fig. 1c) with frequencies between $50 \mathrm{~Hz}$ and $100 \mathrm{~Hz}$. The vibration amplitudes were chosen such that the maximum accelerations $a$ of the plate were between $1.4 \mathrm{~g}$ and $1.8 \mathrm{~g}$. In some experiments, the plate was tilted to introduce an effective gravitational drag on the grains. A metal sheet served as $2 \mathrm{D}$ confinement with a narrow outlet (Fig. 1d) where the passage of active and passive grains could be studied quantitatively. Videos taken with frame rates between 24 fps and 120 fps were recorded and particle positions, orientations and trajectories were extracted.

\section{Single grain motion on a horizontal plane}

On a horizontal plane, the differences between the active and passive grains are particularly obvious when the Mean Squared Displacements (MSD) of capsule motion along their long axis and perpendicular to it are computed. Figure 2 shows these MSD up to a delay of $2 \mathrm{~s}$. During this short time interval, the orientation of the spherocylinders remains nearly preserved. Over long time intervals, the two directions are mixed by the rotational diffusion of the grains. We averaged 10 datasets with more than 1000 frames per dataset. Along the long axes, the active grains propagate in a preferred direction (toward the white, heavier 'head' end) with MSD $\propto(\Delta t)^{2}$. The passive capsules perform Fickian diffusion.

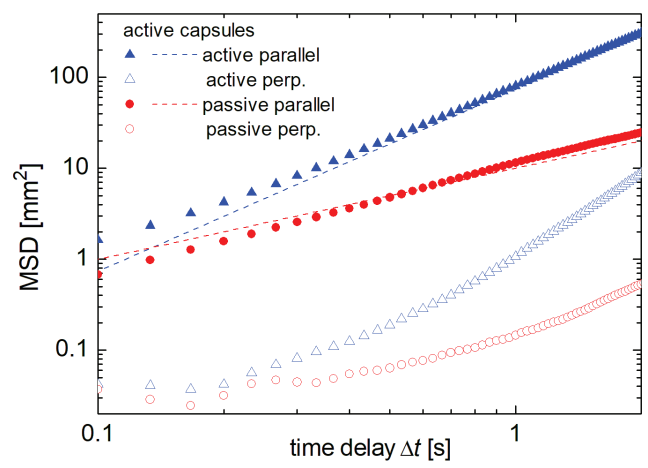

Figure 2. MSD for active and passive capsules on a horizontal plane at $a=1.6 \mathrm{~g}$. The MSD along the long axis follows a $(\Delta t)^{2}$ dependence (blue dashed line) for active grains and $\propto \Delta t$ (red dashed line) for passive ones.

The fixed weights inside the capsules break the rotational symmetry of the grains so that they do not roll sideways. Thus, motion perpendicular to the long axis is slower by one to two orders of magnitude. The reason that the MSD of these displacements are not purely Fickian $(\mathrm{MSD} \propto \Delta t)$ is that we need to define a mean orientation of the capsule in the interval $\Delta t$, which is not exact due to the rotational diffusion [16].

When passive grains reach borders, they are simply reflected. Active grains that reach borders (with their front end) show peculiarities. The silo compartment has vertical walls where active grains colliding with them remain bouncing against the walls head-on. When this happens inside the compartment, the latter may not empty completely during the discharge experiment, few capsules remaining trapped by the silo walls.

The border of the vibrating dish is surrounded by an elevated slanted rim that makes an angle of $\approx 45^{\circ}$ with the plate. This slope obviously inhibits the locking of active grains, they reorient and continue moving (see, however, Sec. 4 on sedimentation patterns).

\section{Gravitaxis and sedimentation on tilted planes}

The passive grains show little effect of a tilt on particle orientations. They sediment in random orientations and only when they reach the bottom barrier of the shaker dish they adopt the energetically most favorable orientation parallel to the border (Fig. 3). Interestingly, the preferential direction is perpendicular to the direction of the effective gravity. This is in contrast to experiments reported by Ramaioli et al. [17], who found that spherocylinders of comparable dimensions aligned normal to the bottom when excited with an acceleration amplitude $a=3 \mathrm{~g}$. In their experiments, the full gravitation force of $1 \mathrm{~g}$ acted in the plane, while in our experiments, the effective gravitation is only $7 \%$ of $g$.
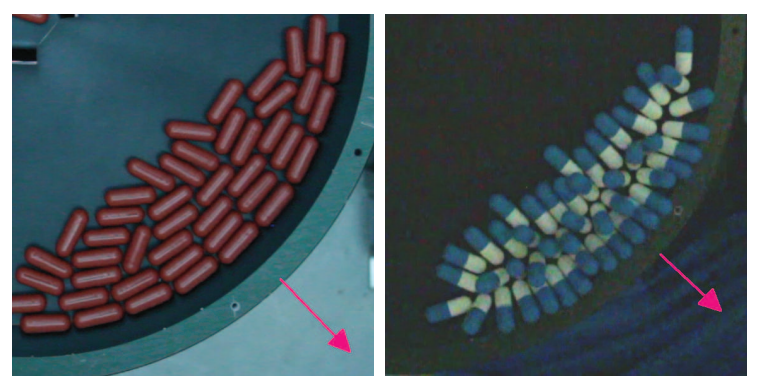

Figure 3. Passive (left) and active (right) grains after sedimentation at the bottom of the vibration dish, $4^{\circ}$ tilt in the direction of the arrows, excitation with $1.6 \mathrm{~g}$.

In contrast to the passive grains, the active capsules show gravitaxis. When moving individually, they tend to orient with the heavier part upward. The reason is that their contact with the vibrating plate is mainly with the heavier (white) end..The lighter blue tail rotates downslope. They develop a certain activity against the gravitational drag (negative gravitaxis), a behavior that is well known in biological systems (e. g. [18]). Individual capsules can occasionally climb upward, or at least reduce their sliding down velocity. The mechanism may be similar to Ref. [19]. When the active grains are in contact and frequently collide with each other, the gravitactic behavior is lost: they settle similar to the passive ones. 
Grains that have passed the orifice move around on the shaker plate, but most of them finally reach the bottom border. Their behavior at that border is completely different from the passive capsules: the first row attaches vertically to the wall, with the heavier parts pointing into the region of the shaker dish. This is intuitively clear, because the lighter tails shift up the rim. The next layer is built by active capsules in opposite orientation (Fig. 3), bouncing with their heavier head end against the first layer of capsules. Inbetween, capsules enclosed between these layers may be pushed the out-of-plane, where they stand up with their heavy end on the shaker dish. This arrangement is stable.

\section{Passage through orifices}

When active and passive grains must pass a narrow outlet forced by an external drag, they behave qualitatively very differently [16]. The most striking observation with the present particle types is that the passage rate $\dot{m}$ (mass $m$ discharged per time) for the passive grains is roughly proportional to the outlet width $d$. This is intuitively clear when one presupposes that all particles pass the outlet with an average velocity independent of the passage width. In contrast, the active capsules show a much stronger $d$ dependence of the discharge rate $\dot{m} \propto d^{2.2}$. For ordinary hopper discharge of spheres, Beverloo's model in 2D predicts a scaling relation $\dot{m} \propto d^{1.5}$ from a dimensional analysis. Thus, the active particles are much more susceptible to the ratio of the orifice width and their own size.

At large excitation strengths, active capsules in the compartment can sometimes lift their tails, while passive capsules remain lying in plane. This not only allows a higher packing fraction in the container, but also facilitates capsule reorientations.

Another interesting aspect is the distribution of delay times $\delta t$ between individual grains passing the bottleneck [20]. The cumulative distribution $P(t>$ $\delta t)$ of delays lasting longer than $\delta t$ can be fitted with a function

$$
P(t>\delta t)=1 /\left(1+\left(\delta t / t_{0}\right)^{\gamma} .\right.
$$

The exponent for active particles, $\gamma_{\mathrm{a}}=1.57$, is substantially smaller than that for passive particles, $\gamma_{p}=2.58$ (Fig. 4), independent of the excitation parameters and plate tilt.

In all discharge experiments, we placed the capsules initially in random orientations. However, elongated grains typically display alignment near the outlet of a discharging hopper [21, 22], an aspect that may be influenced by activity of the grains. In Fig. 5, we analyze the distributions $p(\theta)$ of the capsule orientations directly above the outlet. Averages in (a) and (b) were taken over 10 and 11 experiments, respectively. The compartment was filled with 50 capsules in each run, and data were sampled after the first 10

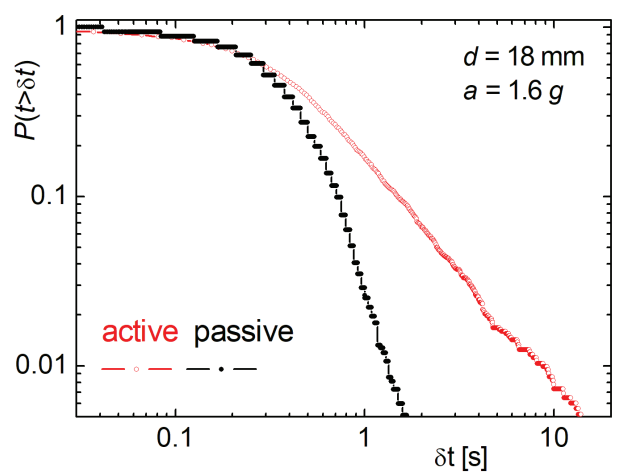

Figure 4. Cumulative distribution of delay times $\delta t$ between subsequent individual particles passing the outlet.
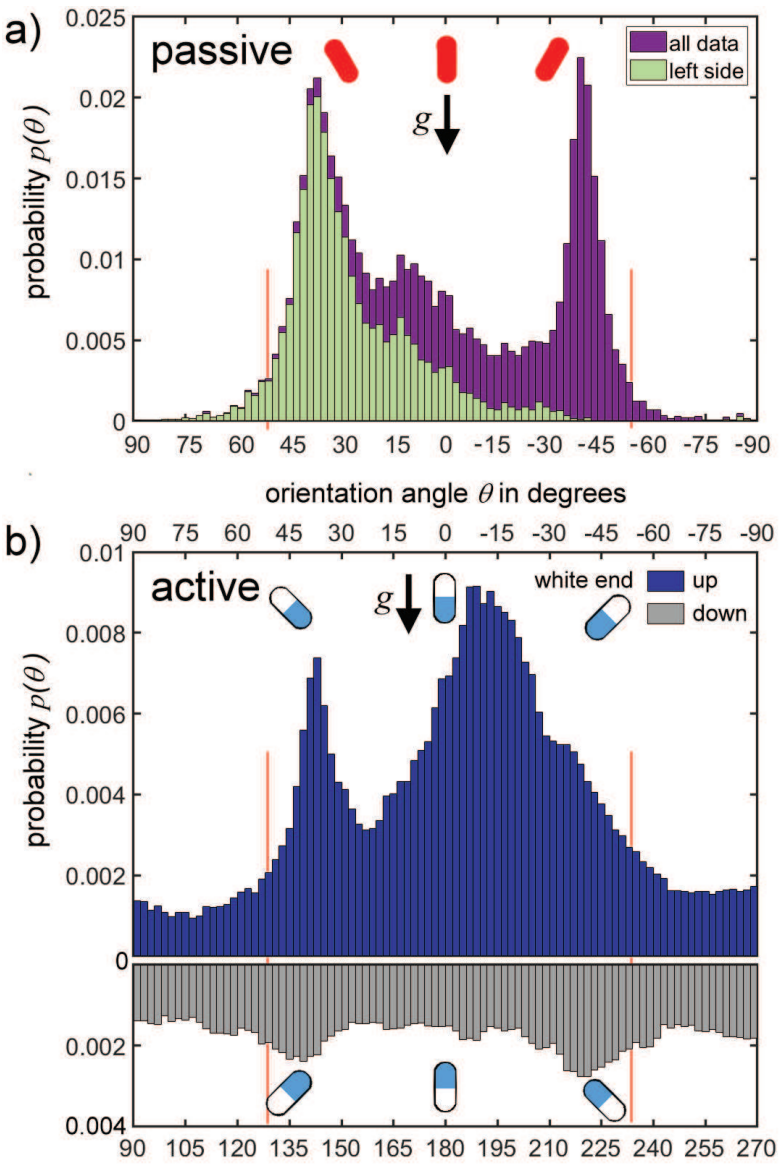

Figure 5. Orientations of capsules in a distance of $\leq$ 1 capsule length above the outlet during discharge, red lines mark the orientation of the compartment borders, $0^{\circ}$ refers to the long axes perpendicular to the outlet (parallel to the direction of gravity), for active particles white head down: (a) passive capsules, probability distribution function of all data (purple) and of capsules lying left of the middle of the outlet (green). (b) active capsules, upper panel: head up, lower panel: head down. Normalization taken over all data. Excitation $a=1.6 \mathrm{~g}$, opening $d=21 \mathrm{~mm}$, tilt $2.5^{\circ}$. 
capsules had passed the orifice. Sampling was stopped when only 5 capsules (a), or 10 capsules (b) remained in the compartment.

Passive capsules display a strong preferential alignment with their long axis roughly parallel to the compartment walls, indicated by the peaks in $p(\theta)$. Particles left of the center of the outlet are preferentially tilted with their upper parts toward the left, and vice verse for capsules in the right half. Note that very few capsules leave aslant, $\left(|\theta|>60^{\circ}\right)$.

The active capsules leave the container in a more random orientation, the alignment with the container walls is still reflected in some peaks in Fig. 5 but less pronounced. Interestingly, capsules with their heavy (white) end upward (blue part of the probability distribution in Fig. 5 b) show much stronger wall alignment than those pointing with their white end down (grey part of $p(\theta)$ ). Notably, $68 \%$ point with their heavier end away from the outlet. The heavy end pointing upward is also the preferred orientation of single active particles on the inclined wall (see Sec. 4).

Out-of-plane flips of the capsules by $180^{\circ}$ were sometimes observed in the direct vicinity of the outlet. It is also obvious that the aslant orientations $\theta<-60^{\circ}, 60^{\circ}<\theta<120^{\circ}$ and $\theta>240^{\circ}$ occur much more frequently for active particles, in contrast to the passive ones.

\section{Summary}

We have studied the dynamic behavior of active and passive spherocylinders on a plane in different situations, starting with the motion on a horizontal plane, where the active grains exhibit a directed propagation in the direction of their long axis, while the passive grains move purely diffusively. On a tilted plane, an effective gravitation acts on the grains, leading to sedimentation. At the same time, active grains experience a torque influencing their orientation, negative gravitaxis is observed. The sedimentation velocity of isolated active grains is reduced compared to passive ones. Active grains may even climb up temporarily.

Finally, we introduced a bottleneck situation to demonstrate the qualitative differences in the passage of active and passive grains through bottlenecks. In the compartment, passive grains are aligned parallel to the container walls. Wall alignment is less pronounced for active grains. The active grains show some gravitactic behavior close to the orifice, preferentially pointing with their heavy ends upward. Understanding the physical mechanism behind the distinct discharge dynamics of active and passive grains requires more systematic studies of the grain packings and dynamics inside the container.

M.M. acknowledges funding by a research Grant of the Iranian Ministry of Science, Research and Technology, and Klaus Kassner for supporting part of her stay in Magdeburg, DLR is acknowledged for funding within project 50WM2048, and DFG for partial support within projects STA $425 / 46$ and HA 8467/2. We thank C. Wagner for lending the shaker.

\section{References}

[1] V. Narayan, S. Ramaswamy, N. Menon, J. Stat. Mech.: Theory Exp. p. P01005 (2006)

[2] V. Narayan, S. Ramaswamy, N. Menon, Science 317, 205 (2007)

[3] A. Kudrolli, G. Lumay, D. Volfson, L. Tsimring, Phys. Rev. Lett. 100, 058001 (2008)

[4] D. Volfson, A. Kudrolli, L. Tsimring, Phys. Rev. E 70, 051312 (2004)

[5] J.C. Tsai, F. Ye, J. Rodriguez, J.P. Gollub, T.C. Lubensky, Phys. Rev. Lett. 94, 214301 (2005)

[6] A. Kudrolli, Phys. Rev. Lett. 104, 088001 (2010)

[7] J. Deseigne, S. Leonard, O. Dauchot, H. Chate, Soft Matter 8, 5629 (2012)

[8] N. Kumar, H. Soni, S. Ramaswamy, A.K. Sood, Nat. Commun. 5, 4688 (2014)

[9] A. Nicolas, M. Kuperman, S. Ibañez, S. Bouzat, C. Appert-Rolland, Sci. Reports 9, 105 (2018)

[10] C. Scholz, M. Engel, T. Pöschel, Nat. Commun. 9, 931 (2018)

[11] C. Gandra, Dynamics of a vibration driven bristle bot (2019), Master Thesis, Clemson University, https://tigerprints.clemson.edu/all_theses/3149

[12] T. Barois, J.F. Boudet, N. Lanchon, J.S. Lintuvuori, H. Kellay, Phys. Rev. E 99, 052605 (2019)

[13] H. Soni, N. Kumar, J. Nambisan, R.K. Gupta, A.K. Sood, S. Ramaswamy, Soft Matter 16, 7210 (2020)

[14] M. Bär, R. Großmann, S. Heidenreich, F. Peruani, Annu. Rev. Cond. Mat. Phys. 11, 441 (2020)

[15] C. Bechinger, R.D. Leonardo, H. Löwen, C. Reichhardt, G. Volpe, G. Volpe, Rev. Mod. Phys. 88, 045006 (2016)

[16] M. Mohammadi et al., New J. Phys. 22, 123025 (2020)

[17] M. Ramaioli, L. Pournin, T. Liebling, Numerical and experimental investigation of alignment and segregation of vibrated granular media composed of rods and spheres, in Powders and Grains (2005), p. 1359

[18] A.M. Roberts, Biol. Bull. 210, 78 (2006)

[19] Z.S. Khan, A. Steinberger, R. Seemann, S. Herminghaus, New J. Phys. 13, 053041 (2011)

[20] I. Zuriguel et al., Scientific Reports 4, 7324 (2014)

[21] T. Börzsönyi et al., New J. Phys. 18, 093017 (2016)

[22] B. Szabo, Z. Kovacs, S. Wegner, A. Ashour, D. Fischer, R. Stannarius, T. Börzsönyi, Phys. Rev. E 97, 062904 (2018) 